\title{
Population Pharmacokinetic Analysis and Exploratory Exposure-Bleeding Rate Relationship of Emicizumab in Adult and Pediatric Persons with Hemophilia A
}

\author{
Sylvie Retout ${ }^{1}$ (D) $\cdot$ Christophe Schmitt ${ }^{1} \cdot$ Claire Petry $^{1} \cdot$ François Mercier $^{1} \cdot$ Nicolas Frey $^{1}$
}

Published online: 5 June 2020

(c) The Author(s) 2020

\begin{abstract}
Background Emicizumab is a bispecific monoclonal antibody developed for routine prophylaxis of bleeding in people with hemophilia A (PwHA). This work characterizes the pharmacokinetics of emicizumab in adult and pediatric PwHA, identifies factors contributing to its between-person variabilities, compares the pharmacokinetics following different dosing regimens, and makes a descriptive assessment of the exposure-bleeding events relationship.

Methods A population pharmacokinetic model was developed, using a database of 389 PwHA from five clinical studies. Potential baseline covariate effects were assessed, including body size, age, race, presence of factor VIII inhibitors, and albumin levels. Using the population pharmacokinetic model, the estimated individual average exposures over the administration period were compared across categories of annualized bleeding rate.

Results A linear one-compartment model with first-order absorption and elimination processes and no lag time best described the emicizumab pharmacokinetics. Body weight, albumin levels, age, and black race were statistically correlated with primary pharmacokinetic parameters, but only body weight had an important influence on exposure. Dosing regimens of $1.5 \mathrm{mg} / \mathrm{kg}$ weekly, $3 \mathrm{mg} / \mathrm{kg}$ every 2 weeks, or $6 \mathrm{mg} / \mathrm{kg}$ every 4 weeks provided similar average concentrations at steady state. A trend for lower exposure was observed in the small proportion of PwHA having an annualized bleeding rate $>4$ (11.9\%), suggesting that reducing exposure to lower levels may potentially increase the bleeding risk.

Conclusions Emicizumab pharmacokinetics in PwHA was described with dose-independent parameters. Body weight was an important predictor of emicizumab pharmacokinetics. All three dosing regimens are predicted to achieve similar exposure associated with clinically meaningful prevention of bleeding.
\end{abstract}

\section{Introduction}

Hemophilia $\mathrm{A}$ is a chromosome $\mathrm{X}$-linked recessive deficiency of factor VIII (FVIII) activity resulting in lifelong bleeding. Severe disease (FVIII $<1 \mathrm{IU} / \mathrm{dL}$ ) is accompanied by high morbidity and mortality due to recurrent frequent spontaneous bleeds into joints, muscles, or soft tissues. Treatment relies on either episodic intravenous (IV) infusion of FVIII concentrates in response to symptoms

Electronic supplementary material The online version of this article (https://doi.org/10.1007/s40262-020-00904-z) contains supplementary material, which is available to authorized users.

Sylvie Retout

sylvie.retout@ roche.com

1 Roche Pharma Research and Early Development, Pharmaceutical Sciences, Clinical Pharmacology, Roche Innovation Center Basel, Postfach, 4070 Basel, Switzerland of a bleed or scheduled prophylactic FVIII administration in an effort to prevent bleeds from occurring. Factor VIII prophylaxis has been proven to minimize bleeding events and complications [1]; however, it requires lifelong IV infusion two to four times each week, resulting in high treatment burden, unsatisfactory adoption, incomplete adherence, and complications associated with indwelling IV catheters [2]. Up to $30 \%$ of persons with hemophilia A (PwHA) develop neutralizing alloantibodies (inhibitors) against infused FVIII [3], and those who express hightiter inhibitors are treated with bypassing agents, such as activated prothrombin complex concentrate [4] or recombinant activated factor VII [5], which have decreased efficacy relative to FVIII.

Emicizumab (Hemlibra ${ }^{\circledR}$; F. Hoffmann-La Roche, Ltd, Basel, Switzerland) is a humanized bispecific monoclonal antibody that bridges activated factor IX and factor X [6], thereby restoring the function of missing activated FVIII needed for effective hemostasis at the site of bleeding in 


\section{Key Points}

Pharmacokinetics of emicizumab was characterized in adults, adolescents, and children with hemophilia A.

Maintenance doses of $1.5 \mathrm{mg} / \mathrm{kg}$ weekly, $3 \mathrm{mg} / \mathrm{kg}$ every 2 weeks, or $6 \mathrm{mg} / \mathrm{kg}$ every 4 weeks are expected to provide similar exposure associated with clinically meaningful prevention of bleeding; lower dose (exposure) could potentially lead to a lower effect.

Apart from the body-weight dosing, no requirements for additional dose adjustment were identified.

PwHA. It has no structural relationship to FVIII and does not induce or enhance the development of inhibitors to FVIII or to other coagulation factors [7]. Emicizumab is administered subcutaneously with high bioavailability [8], obviating the need for venous access. Its long elimination half-life $\left(t_{1 / 2}\right)$, of 4-5 weeks [9], enables weekly or less frequent dosing, which is an advantage over frequent IV administration of FVIII therapies [10]. Emicizumab may therefore substantially improve upon current treatment options for PwHA and fulfill a strong medical need.

Three different subcutaneous (SC) emicizumab dosing regimens, $3 \mathrm{mg} / \mathrm{kg}$ once weekly (QW) for 4 weeks followed by: $1.5 \mathrm{mg} / \mathrm{kg}(\mathrm{QW}), 3 \mathrm{mg} / \mathrm{kg}$ every 2 weeks (Q2W), or $6 \mathrm{mg} / \mathrm{kg}$ every 4 weeks (Q4W), were confirmed in recent trials [11-14] to have an acceptable safety profile and similar clinically meaningful efficacy in preventing or reducing the frequency of bleeding episodes in PwHA with or without FVIII inhibitors. In addition, the first characterization of emicizumab pharmacokinetics was performed on phase I/ II data on a limited number of people [15].

The aims of the presented analyses were to: (i) develop a population pharmacokinetic (popPK) model to characterize the pharmacokinetics of emicizumab in PwHA enrolled in phase I-III clinical studies, and to identify factors that contribute to its between-person variability (BPV); (ii) compare the emicizumab PK time course following the QW, Q2W, or Q4W dosing regimens; and (iii) make a descriptive assessment of the relationship between emicizumab exposure and the rate of bleeding events over a year interval, to support the dosing recommendation.

\section{Materials and Methods}

\subsection{Clinical Studies and Patient Data}

The popPK analysis population consisted of 389 pediatric, adolescent, or adult PwHA with or without inhibitors against
FVIII who were participants in one of five clinical studies (a phase I/II study or four phase III: HAVEN 1-4; Table 1). All PwHA included in this analysis were emicizumab treatment naïve before study entry, then were treated with emicizumab, and had emicizumab plasma concentration data available.

All study protocols were approved by the institutional review board at each site. Participants provided written informed consent before enrollment in the studies, which were conducted in full conformance with the Declaration of Helsinki.

Plasma emicizumab concentrations were measured using a validated bridging enzyme-linked immunosorbent assay. In brief, emicizumab was captured by a rabbit anti-idiotype monoclonal antibody against factor X-binding antigen-binding fragment and detected by a mouse anti-idiotype monoclonal antibody against activated factor IX-binding antigenbinding fragment, followed by a peroxidase-labeled goat anti-mouse immunoglobulin G. The lower limit of quantification was $50 \mathrm{ng} / \mathrm{mL}$ in the phase I/II study and $100 \mathrm{ng} / \mathrm{mL}$ in the phase III studies.

In the phase III HAVEN studies, bleeding event data were recorded by participants/caregivers using an electronic handheld device [11-14]. Treated bleeds, as defined in the HAVEN studies $[11,16]$, were summarized at the patient level into an annualized bleeding rate (ABR) using the following formula:

$\mathrm{ABR}=\frac{\text { Number of treated bleeds }}{\text { Total number of days during the efficacy period }} \times 365.25$.

The reported ABR in this article focuses on treated bleeds only, in line with the primary endpoints of the HAVEN studies $[11,16]$. The start of the efficacy period for each PwHA receiving emicizumab coincided with the day of the first emicizumab dose. The end of the efficacy period was defined as the date of the clinical cut-off or withdrawal from the study, whichever occurred earlier. For PwHA whose dose was up-titrated, the efficacy period ended 1 day prior to the first day of the up-titrated dose.

\subsection{Population Pharmacokinetic Modeling}

\subsubsection{Base Pharmacokinetic Model Development}

Analysis of the PK data was conducted using non-linear mixed-effects modeling with NONMEM (version 7.2.0, FOCE Interaction). In line with the low affinity of emicizumab to its target antigens (activated factor IX and factor $\mathrm{X})$, and observations from the phase I/II study $[6,17,18]$, emicizumab was assumed to have linear pharmacokinetics without target-mediated drug disposition. Models with one and two compartments, with a first-order absorption with or without lag time, parameterized in terms of clearance(s) 


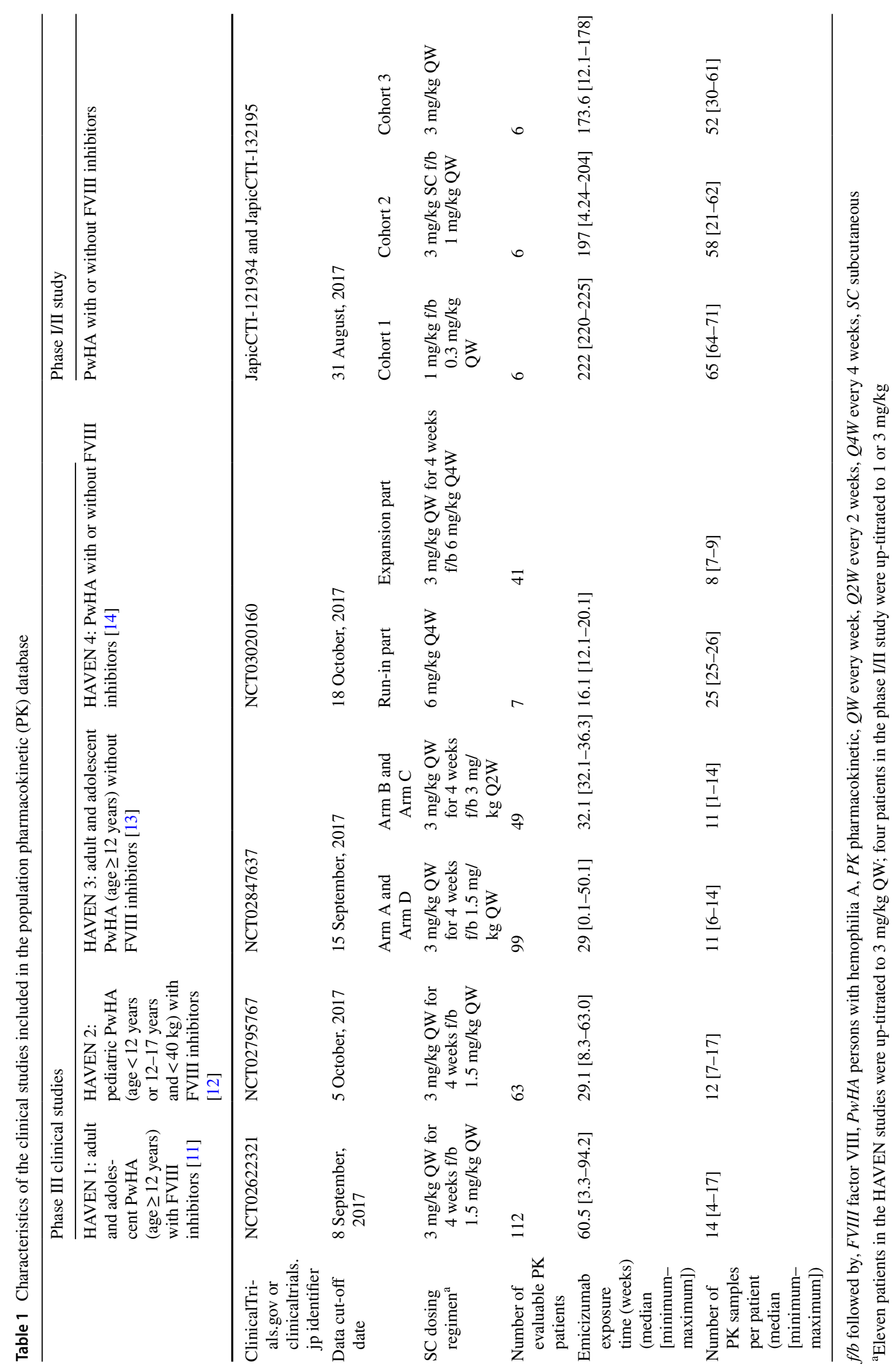


and volume(s) of distribution, were tested. Between-person variability was modeled, assuming a log-normal distribution of the individual parameters. The residual error model was assumed combined, with an additive and multiplicative part.

\subsubsection{Covariate Model Development}

The potential association between patient covariates and all of the primary PK parameters were evaluated in a stepwise manner. First, a generalized additive modeling (GAM) [19], implemented in Xpose (version 4.6.0), was conducted, regressing the inter-individual random-effect estimates on the individual covariate values. Possible covariate relationships were retained if they showed a reduction of at least 4 points on the Akaike information criterion [20] and a total inclusion frequency $\geq 0.8$ from a bootstrap of the GAM [21].

The retained covariates were further tested simultaneously on all parameters in a full covariate model [22-24], after checking their scientific relevance and/ or mechanistic plausibility. Continuous covariates were normalized to the population median values, using the general equations: $\theta_{i}=\theta_{\mathrm{pop}} \cdot\left(\operatorname{cov}_{i} / \operatorname{cov}_{m}\right)^{\theta_{\mathrm{cov}}}$ or $\theta_{i}=\theta_{\text {pop }} \cdot\left(1+\theta_{\text {cov }} \cdot\left(\operatorname{cov}_{i}-\operatorname{cov}_{m}\right)\right)$, where $\theta_{i}$ is the individual model-predicted $\mathrm{PK}$ parameter for an individual with a covariate value, $\operatorname{cov}_{i}, \theta_{\text {pop }}$ is the population central tendency for the PK parameter $\theta, \operatorname{cov}_{m}$ is the population median or widely used standard value of the covariate, and $\theta_{\text {cov }}$ is the covariate effect. The more appropriate of the two equations was selected based on visual inspection of the individual parameters with respect to the covariate.

Categorical covariates were tested and incorporated in the model as index variables using the general equation: $\theta_{i}=\theta_{\text {pop }} \cdot\left(1+\theta_{\text {cov }} \cdot\left(\operatorname{cov}_{i}\right)\right)$, where $\operatorname{cov}_{i}$ is coded as either 0 or 1 . To investigate the combined effects of covariates on a given PK parameter, the covariate effect parts from the three equations above were multiplied.

Tested covariates at baseline included age (pediatric PwHA: $\geq 1$ to $<12$ years, adolescent: $\geq 12$ years to $<18$ years, and adult PwHA: $\geq 18$ years), body mass index, body weight (BW), body surface area, race (white, black, Asian [including Japanese], other, or unknown), patient status (with or without FVIII inhibitors), and albumin (ALB) level. Summary statistics of these covariates are reported in Tables 2 and 3.

Inferences about the importance for the pharmacokinetics of the estimated covariate effects were made based on the magnitude and precision of covariate parameter estimates. A covariate effect was defined as having "no important impact on the pharmacokinetics" if the entire $90 \%$ interval of posterior distribution of 10,000 sampled estimates of the effect fell within the covariate effect unimportant region. This region was defined as $\pm 20 \%$ of the median sampled typical parameter normalized to the median [25]. The full model was then reduced, dropping covariate effects that were not statistically significant $(95 \%$ confidence interval including the null value) and therefore deemed to be not important.

The influence of renal and hepatic impairment on the pharmacokinetics of emicizumab was investigated using descriptive statistics. People were classified into normal, mild, moderate, and severe categories. For renal function, the classification used was that of either the National Kidney Foundation [26] based on the Cockcroft-Gault formula [27] at baseline (HAVEN 1, HAVEN 3, and HAVEN 4) or the Bedside Schwartz and Work formula [28] at baseline (HAVEN 2). For hepatic function, the National Cancer Institute Organ Dysfunction Working Group's hepatic impairment categorization [29] was followed. Inter-individual random-effect estimates were compared across the different patient categories.

\subsubsection{Model Selection and Evaluation Criteria}

The ability of the investigated popPK models to describe the observed data was assessed and compared using multiple criteria. First, a decrease in the objective function value of at least 6.63 was considered statistically significant. This corresponds to a nominal $p<0.01$ and 1 degree of freedom in the Chi-square distribution of the difference in objective

Table 2 Summary (median [minimum-maximum]) of the continuous covariates included in the population pharmacokinetic database

\begin{tabular}{|c|c|c|c|c|c|c|}
\hline & All studies & HAVEN 1 & HAVEN 2 & HAVEN 3 & HAVEN 4 & $\begin{array}{l}\text { Japanese phase I/ } \\
\text { II study }\end{array}$ \\
\hline $\begin{array}{l}\text { Number of PwHA with } \\
\text { evaluable PK }\end{array}$ & 389 & 112 & 63 & 148 & 48 & 18 \\
\hline Age (years) & $30.0[1.22-77.0]$ & $28.5[12.0-75.0]$ & $7.12[1.22-15.7]$ & $38.0[13.0-77.0]$ & $38.0[14.0-68.0]$ & $30.0[12.0-58.0]$ \\
\hline Body weight (kg) & $69.1[9.50-156]$ & $71.7[40.1-156]$ & $22.6[9.50-63.0]$ & 76.6 [43.0-139] & $74.2[43.3-102]$ & $60.4[40.8-81.7]$ \\
\hline $\begin{array}{l}\text { Body mass index }(\mathrm{kg} / \\
\left.\mathrm{m}^{2}\right)\end{array}$ & $23.6[13.1-52.4]$ & $23.9[15.1-52.4]$ & $16.2[13.1-30.3]$ & $25.0[16.8-40.6]$ & 23.7 [16.9-33.2] & $22.5[14.4-30.0]$ \\
\hline Body surface area $\left(\mathrm{m}^{2}\right)$ & $1.81[0.479-2.58]$ & $1.83[1.31-2.58]$ & 0.86 [0.469-1.69] & $1.93[1.41-2.58]$ & 1.85 [1.41-2.17] & $1.67[1.40-1.98]$ \\
\hline Albumin level (g/L) & $45.0[16.8-56.6]$ & $45.0[33.0-54.9]$ & $43.0[16.8-51.1]$ & $46.0[37.0-56.6]$ & $45.5[34.0-56.0]$ & $45.5[42.0-52.0]$ \\
\hline
\end{tabular}

$P w H A$ persons with hemophilia A, $P K$ pharmacokinetics 


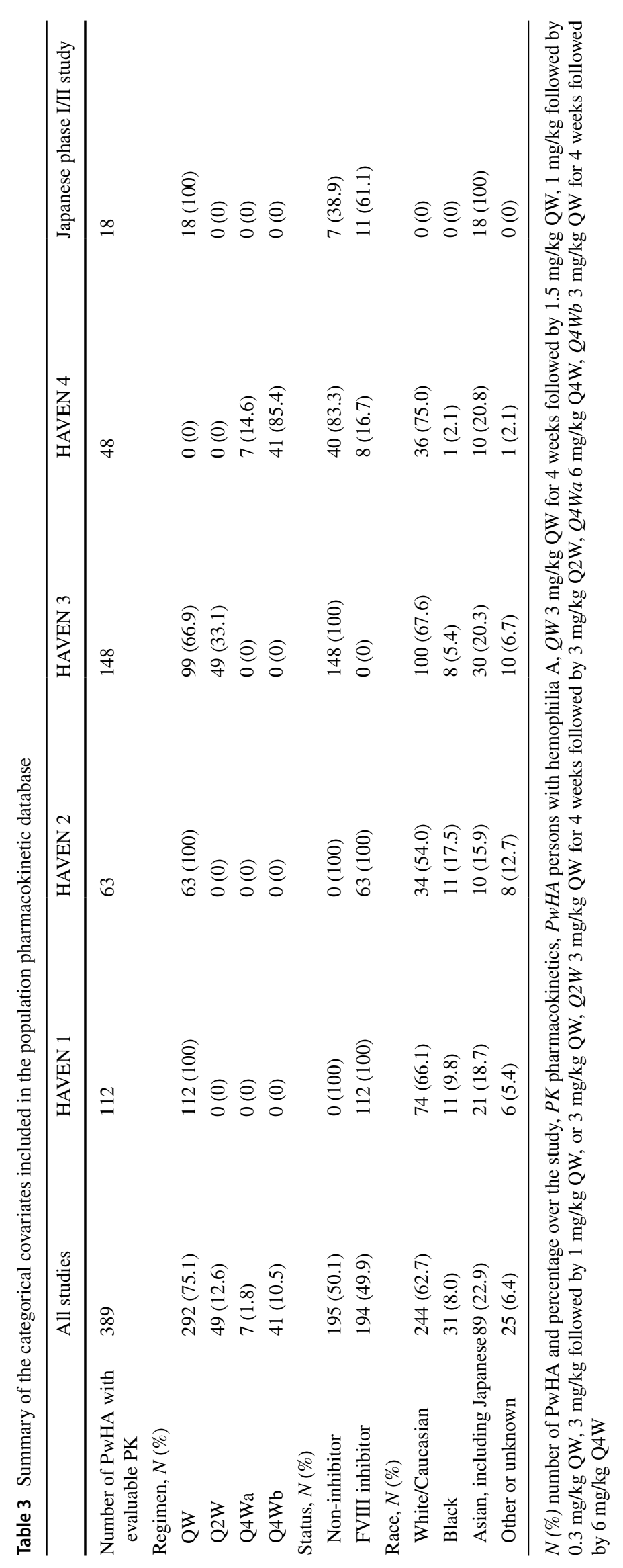


function value between hierarchical models. Standard goodness-of-fit plots were also used as criteria, together with the inspection of the relative standard errors of the parameters. Shrinkage was computed for each individual parameter to evaluate the degree of regression to the mean value. Once selected, the predicted performances of the final model were evaluated using predicted-corrected visual predictive checks [30] for all data or data split per study and/or dosing regimen, where relevant.

\subsection{Pharmacokinetic Model-Based Simulations}

Individual primary PK parameter estimates were obtained for each PwHA from the final popPK model, and were used to simulate individual secondary PK parameters for the three dosing regimens: $1.5 \mathrm{mg} / \mathrm{kg} \mathrm{QW}, 3 \mathrm{mg} / \mathrm{kg}$ Q2W, and $6 \mathrm{mg} /$ $\mathrm{kg}$ Q4W. For each of them, steady-state PK profiles were simulated by steps of $6 \mathrm{~h}$, and individual trough concentration $\left(C_{\text {trough,SS }}\right)$ and maximum concentration $\left(C_{\max , \mathrm{SS}}\right)$ at steady state were computed as the minimum and maximum simulated values, respectively; the corresponding time to reach $C_{\max , \mathrm{SS}}\left(t_{\max , \mathrm{SS}}\right)$ was also reported. Secondary PK parameters, including elimination $t_{1 / 2}$, absorption half-life, area under the curve of plasma concentration over a dosing interval $\tau$ at steady state $\left(\mathrm{AUC}_{\mathrm{SS}, \tau}\right)$ with $\tau=1,2$, or 4 weeks, average concentration $\left(C_{\mathrm{av}, \mathrm{SS}}\right.$, calculated as $\left.\mathrm{AUC}_{\mathrm{SS}, \tau} / \tau\right)$, and peak-to-trough ratio at steady state $\left(C_{\mathrm{max}, \mathrm{SS}} / C_{\text {trough,SS }}\right)$, were also derived and summarized together with $C_{\text {trough,SS }}$ and $C_{\text {max,Ss}}$.

Additional simulations were also conducted to investigate the impact of the retained covariates on the steadystate PK profile, and to graphically display the predicted PK profile over the first 6 months of treatment in children (age $<12$ years) and adults and adolescents (age $\geq 12$ years), for the three different dosing regimens. For these PK profile simulations, 1000 virtual PwHA were created. Covariates were sampled, with replacement, in the popPK database, keeping the link at the individual level between the covariate values. Summaries of the simulated PK profiles were then computed using the median and the 5th and the 95th percentiles.

\subsection{Graphical Exploratory Analysis of Exposure- Bleeding Rate Relationships for People with Hemophilia A}

Graphical exploratory analyses were conducted to investigate whether the variability in emicizumab exposure could explain the variability in the ABR, using data from the four phase III studies at the tested QW, Q2W, or Q4W dosing regimens. Because PwHA in those studies were not treated for the same duration at the time of the data cut-off, only efficacy data of PwHA from the PK database and who were treated for at least 24 weeks were considered. In addition, the data cut-off date of HAVEN 4 was extended from 18 October, 2017 to 15 December, 2017, and included the 41 PwHA in the expansion cohort (Q4W dosing regimen).

The graphical exposure-efficacy analyses compared the estimated individual average concentration $\left(C_{\mathrm{av}}\right)$ distribution across PwHA grouped by severity of their respective estimated ABRs, as defined in Sect. 2.1: $\mathrm{ABR}=0, \mathrm{ABR}$ in $>0$ to 4 , and $\mathrm{ABR}>4$. These $C_{\mathrm{av}}$ were defined as the estimated total exposure over the period of dose administration before any up-titration divided by the length of that period.

\section{Results}

\subsection{Emicizumab Population Pharmacokinetic Model}

A total of 4966 plasma concentrations collected from 383 evaluable PwHA pharmacokinetic profiles (Fig. S1 of the Electronic Supplementary Material [ESM]) were used for the base popPK model development. Data below the lower limit of quantification were limited $(<1 \%$ of the data) and therefore excluded from the analysis. A one-compartment model with first-order absorption and first-order elimination, and without any lag time, best described the data, as previously reported for the phase I/II study [15]. The primary PK model parameters estimated were apparent clearance (CL/F), apparent volume of distribution $(V / F)$, and absorption rate constant. Between-person variability was incorporated on all of the primary parameters. The additive part of the residual error model was fixed to the half of the lower limit of quantification in the phase I/II study; the multiplicative part was estimated.

Body weight was strongly positively correlated with CL/F and $V / F$, and was included in the base model, with estimated power coefficients of 0.939 and 1.07 for CL/F and $V / F$, respectively. This reduced the BPV on CL/ $F$ from 56.4 to $30.0 \%$ and on $V / F$ from 60.7 to $28.1 \%$. Eta shrinkage was modest for CL/F and $V / F$ ( $4.1 \%$ and $8.8 \%$, respectively), limiting the risk of misinterpretation of the GAM analysis [31]; it was larger for absorption rate constant (42.7\%).

Two PwHA with abnormally low ALB at baseline (16.8 g/L and $27.0 \mathrm{~g} / \mathrm{L}$, respectively), compared to the normal range for ALB: $37-55 \mathrm{~g} / \mathrm{L}$ [32], were removed from the covariate model development, therefore conducted using data from $381 \mathrm{PwHA}$. From the GAM analysis, the covariates selected to be further investigated in NONMEM were age and ALB on CL/F, and age and race (black) on $\mathrm{V} / \mathrm{F}$. Because age was found to impact the two apparent parameters $\mathrm{CL} / \mathrm{F}$ and $\mathrm{V} / \mathrm{F}$ in a similar manner, an additional investigation of the impact of age on the apparent bioavailability parameter $(\mathrm{F})$ was performed. No covariates were selected to be further tested on absorption rate constant. 
Table 4 NONMEM parameter estimates for the final population pharmacokinetic model

\begin{tabular}{|c|c|c|c|c|c|}
\hline Parameter & Unit & Estimate & $\operatorname{RSE}(\%)$ & 95\% CI (lower-upper) & Shrinkage $(\%)$ \\
\hline \multicolumn{6}{|c|}{ Fixed effects (BW $70 \mathrm{~kg}$; ALB $45 \mathrm{~g} / \mathrm{L}$; age $<30$ years) } \\
\hline $\mathrm{CL} / F$ & L/day & 0.272 & 1.9 & $0.262-0.282$ & \\
\hline$V / F$ & $\mathrm{~L}$ & 10.4 & 1.9 & $10.0-10.8$ & \\
\hline KA & 1/day & 0.536 & 7.1 & $0.462-0.610$ & \\
\hline \multicolumn{6}{|l|}{ Inter-individual variability ${ }^{\mathrm{a}}$} \\
\hline $\mathrm{CL} / F$ & $\mathrm{CV} \%$ & 28.7 & $8.6^{\mathrm{b}}$ & & 3.7 \\
\hline$V / F$ & $\mathrm{CV} \%$ & 25.9 & $8.9^{\mathrm{b}}$ & & 10.3 \\
\hline KA & $\mathrm{CV} \%$ & 72.5 & $14.7^{\mathrm{b}}$ & & 40.6 \\
\hline Correlation $\mathrm{CL} / \mathrm{F}-V / F$ & - & 0.217 & $31.8^{\mathrm{c}}$ & & \\
\hline Correlation $\mathrm{CL} / F-\mathrm{KA}$ & - & -0.341 & $25.0^{\mathrm{c}}$ & & \\
\hline \multicolumn{6}{|l|}{ Covariate effects } \\
\hline Effect of BW on CL/F & - & 0.911 & 3.2 & $0.854-0.968$ & \\
\hline Effect of ALB on CL/F & - & $1.57 \times 10^{-2}$ & 28.4 & $0.696 \times 10^{-2}$ to $2.44 \times 10^{-2}$ & \\
\hline Effect of $\mathrm{BW}$ on $\mathrm{V} / \mathrm{F}$ & - & 1.00 & 3.0 & $0.941-1.06$ & \\
\hline Effect of Black on V/F & - & -0.215 & 19.7 & -0.298 to -0.132 & \\
\hline Effect of AGE $>30$ years on $F$ & - & $6.51 \times 10^{-3}$ & 16.3 & $4.43 \times 10^{-3}$ to $8.59 \times 10^{-3}$ & \\
\hline \multicolumn{6}{|l|}{ Error model $^{\mathrm{d}}$} \\
\hline$\sigma_{1}$ (additive) & $\mu \mathrm{g} / \mathrm{mL}$ & 0.025 Fix & - & & \\
\hline$\sigma_{2}$ (proportional) & $\%$ & 14.6 & 2.0 & $14.0-15.2$ & \\
\hline Condition number ${ }^{\mathrm{e}}$ & - & 13.7 & - & - & - \\
\hline
\end{tabular}

$\sigma$ residual error, $A G E$ age, $A L B$ albumin level, $B P V$ between-patient variability, $B W$ body weight, $C I$ confidence interval, $C L / F$ apparent clearance, $C V$ coefficient of variation, $F$ apparent bioavailability parameter, Fix fixed, $K A$ absorption rate constant, $P K$ pharmacokinetic, $R S E$ relative standard error of estimate, $V / F$ apparent volume of distribution

${ }^{\mathrm{a}}$ Assumed to follow an exponential error model

${ }^{\mathrm{b}} \mathrm{RSE}$ computed for the corresponding variance

${ }^{\mathrm{c}} \mathrm{RSE}$ computed for the corresponding covariance

${ }^{\mathrm{d}}$ Assumed to follow a combined additive-plus-proportional error model

${ }^{\mathrm{e}}$ Ratio of the largest eigenvalue to the smallest eigenvalue of the correlation matrix

The full covariate approach retained the effects of BW and ALB on CL/F, BW and black race on V/F, and age on the F. Other covariates did not have a significant effect on the PK parameters.

The parameter estimates of the final popPK model are reported in Table 4 and the diagnostic plots in Fig. 1 (also Fig. S2 of the ESM for additional investigations between parameters and the retained covariates). Precise estimates were achieved and this final model did not present any major deficiencies in the goodness-of-fit plots. The equations for the covariate models are presented below:

$\mathrm{CL} / F=0.272 \times(\mathrm{BW} \div 70)^{0.911} \times(1-0.0157 \times(\mathrm{ALB}-45))$,

$V / F=10.4 \times(\mathrm{BW} \div 70)^{1.00} \times(1-0.215 \times \mathrm{BLK})$,

$\mathrm{IF}(\mathrm{AGE} \leq 30) F=1$ else $F=1-0.00651 \times($ AGE -30$)$,

where BLK is 1 if persons are black, and 0 otherwise.
The importance of the detected covariate effects on the PK parameters is represented in Fig. 2. Body weight strongly impacts $\mathrm{CL} / \mathrm{F}$ and $V / F$, modifying their typical value up to $-85 /+108 \%$ and $-87 \% /+123 \%$, respectively, for extreme BW values of $9 \mathrm{~kg}$ and $156 \mathrm{~kg}$. Age also strongly reduces $F$ in PwHA > 65 years. In contrast, the effects of ALB on CL/F and of black race on $V / F$ were minimal.

Figure 3 reports predicted-corrected visual predictive checks in different sub-groups of studies (HAVEN 1, HAVEN 2, HAVEN 3, and the expansion part of HAVEN 4), dosing regimens, or ages. Additional predicted-corrected visual predictive checks for the phase I/II study and the runin part of HAVEN 4 are reported in Fig. S3 of the ESM. The final popPK model adequately captures both the central tendency and the BPV of emicizumab pharmacokinetics in the target population, whatever the age group (adolescents/adults, pediatric $\geq 1$ to $<6$ years, or pediatric $\geq 6$ to $<12$ years), the dosing regimen $(\mathrm{QW}, \mathrm{Q} 2 \mathrm{~W}$, or $\mathrm{Q} 4 \mathrm{~W})$, 
Fig. 1 Goodness-of-fit plots for the final pharmacokinetic model for emicizumab. CWRES (IWRES) conditional (individual) weighted residual values, $D V$ observed emicizumab concentrations $[\mu \mathrm{g} / \mathrm{mL}]$, PRED (IPRED) NONMEM predicted emicizumab concentrations $[\mu \mathrm{g} / \mathrm{mL}]$ based on population (individual) pharmacokinetic parameters, Time time after first drug intake (days)
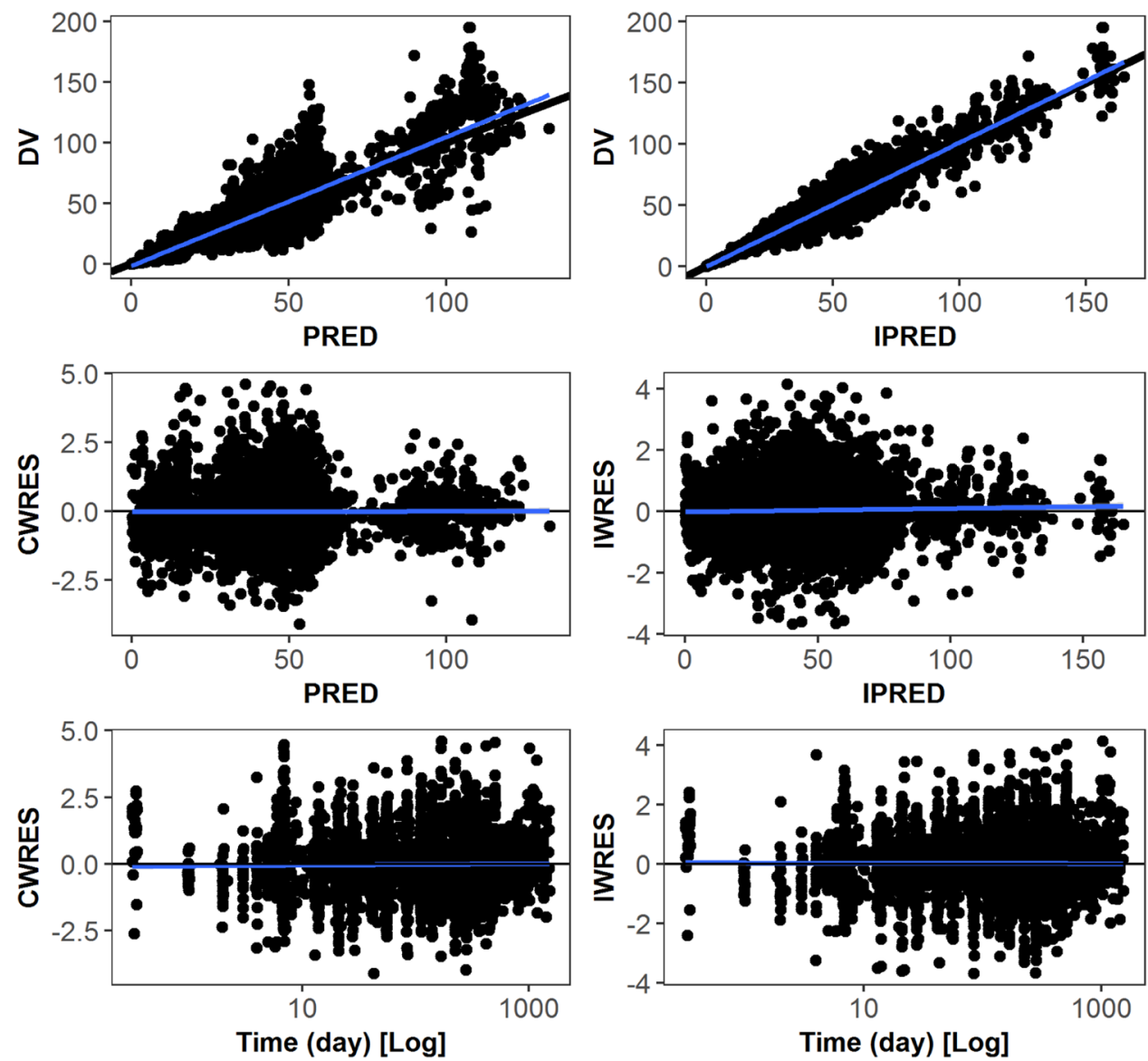

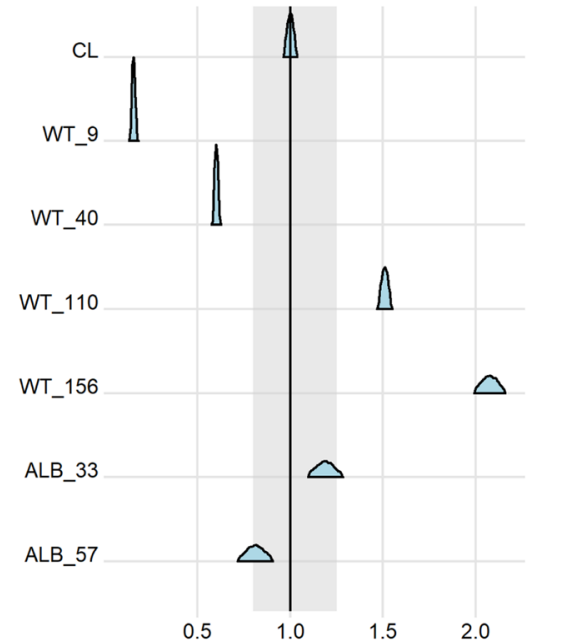

Change in CL/F Relative to Reference Patient

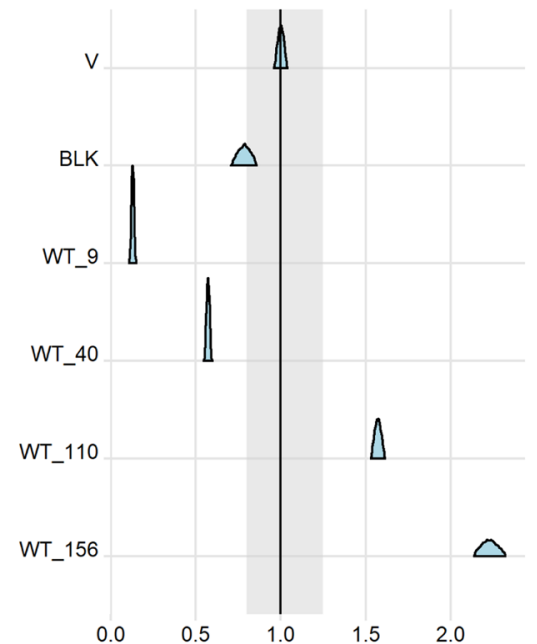

Change in V/F Relative to Reference Patient

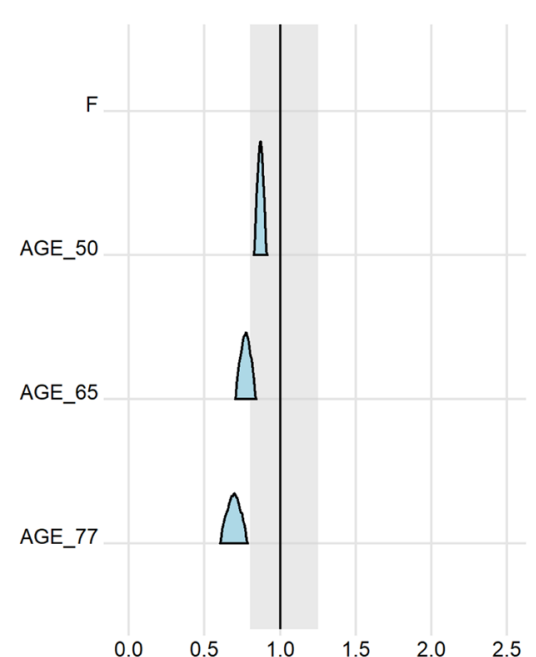

Change in F Relative to Reference Patient
Fig. 2 Effects of the retained covariates on the population pharmacokinetic parameters in the final model. The ratio posterior distributions of the population CL/F (left), V/F (middle), and F (right), calculated for various covariate values over the population parameter value calculated for a reference patient with body weight of $70 \mathrm{~kg}$, albumin (ALB) level of $45 \mathrm{~g} / \mathrm{L}$, and age of 30 years are presented. Selected covariate values include the respective minimum and maximum covariate values observed in the population pharmacokinetic database, as well as arbitrary selected values for illustration purposes. The shaded area represents typical values $\pm 20 \%$ and represents the zone where the covariate effect is not considered potentially important. $A G E \_50, A G E \_65$, and $A G E \_77$ age of 50,65, and 77 years, $A L B \_33$ ALB level of $33 \mathrm{~g} / \mathrm{L}, A L B \_57$ ALB level of $57 \mathrm{~g} / \mathrm{L}, B L K$ black race, $C L \mathrm{CL} / \mathrm{F}$ (L/day), $C L / F$ apparent clearance, $F$ apparent bioavailability parameter, $V$ V/F (L), V/F apparent volume of distribution, WT_9 body weight of $9 \mathrm{~kg}, W T \_40$ body weight of $40 \mathrm{~kg}, W T \_110$ body weight of $110 \mathrm{~kg}, W T \_156$ body weight of $156 \mathrm{~kg}$ 

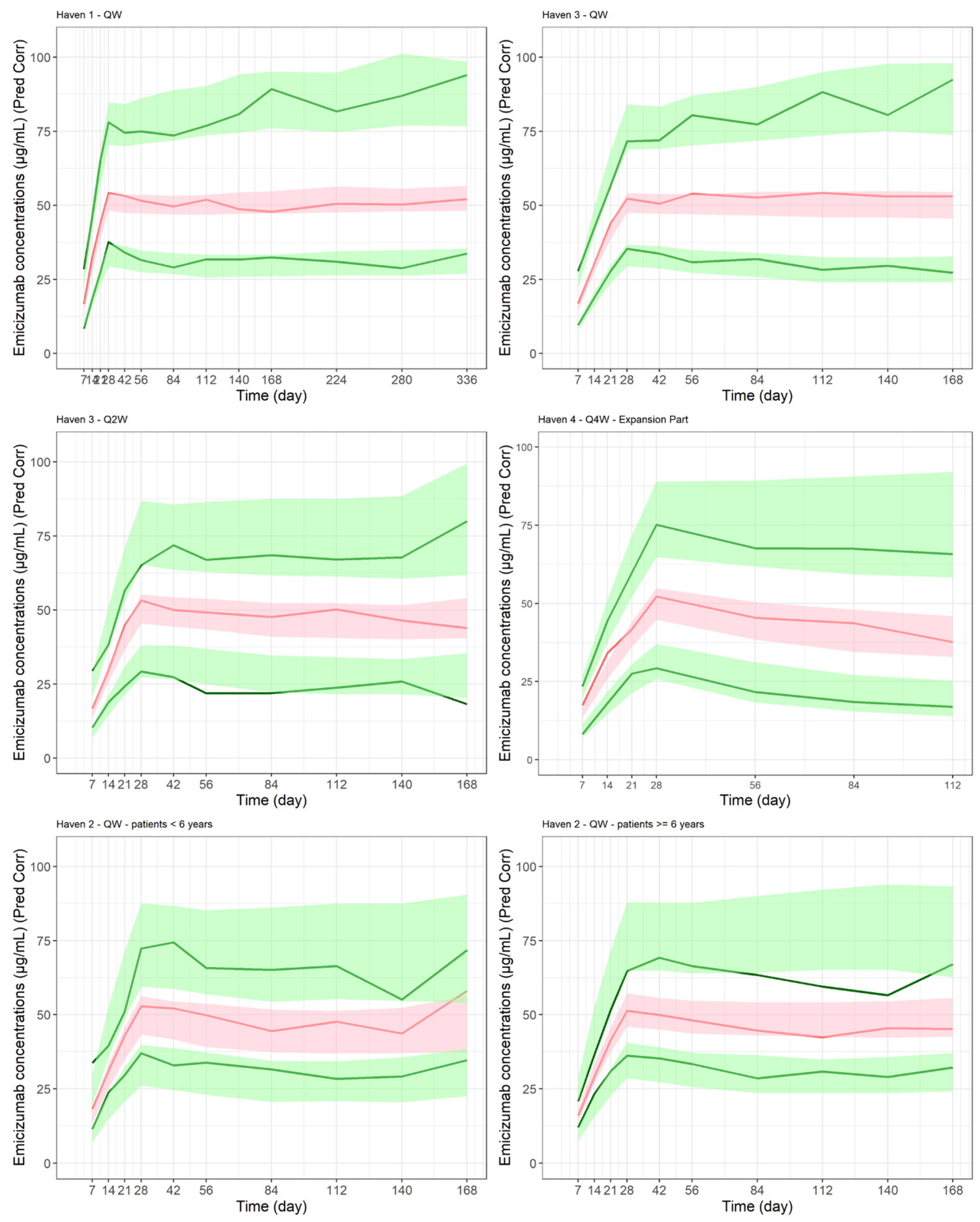

- Observed 5th \& 95th percentiles - Observed median

Fig. 3 Visual posterior predictive check for emicizumab per study, dosing regimen, and age: every week (QW) dosing regimen for HAVEN 1 (top left) and HAVEN 3 (top right); every 2 weeks (Q2W) dosing regimen for HAVEN 3 (middle left); every 4 weeks (Q4W)

\section{$90 \% \mathrm{Cl}$ around simulated 5 th \& 95th percentiles} $90 \% \mathrm{Cl}$ around simulated median dosing regimen in the expansion cohort of HAVEN 4 (middle right); QW dosing regimen in HAVEN 2, persons with hemophilia A (PwHA) $<6$ years (bottom left) and $\geq 6$ years (bottom right). CI confidence interval, Pred Corr predicted-corrected 
Table 5 Secondary pharmacokinetic (PK) parameters derived for emicizumab per considered dosing regimens using the primary individual PK parameters obtained by the final population PK model

\begin{tabular}{|c|c|c|c|c|c|c|}
\hline \multirow[t]{2}{*}{ Secondary PK parameters } & \multicolumn{2}{|c|}{$1.5 \mathrm{mg} / \mathrm{kg} \mathrm{QW}$} & \multicolumn{2}{|c|}{$3 \mathrm{mg} / \mathrm{kg} \mathrm{Q} 2 \mathrm{~W}$} & \multicolumn{2}{|l|}{$6 \mathrm{mg} / \mathrm{kg} \mathrm{Q} 4 \mathrm{~W}$} \\
\hline & Mean (SD) & $\begin{array}{l}\text { Median [5th-95th] } \\
\text { percentiles }\end{array}$ & Mean (SD) & $\begin{array}{l}\text { Median [5th-95th] } \\
\text { percentiles }\end{array}$ & Mean (SD) & $\begin{array}{l}\text { Median [5th-95th] } \\
\text { percentiles }\end{array}$ \\
\hline$t_{1 / 2}($ day $)$ & $26.8(9.16)$ & $25.1[13.9-41.4]$ & $26.8(9.16)$ & 25.1 [13.9-41.4] & $26.8(9.16)$ & $25.1[13.9-41.4]$ \\
\hline$t_{1 / 2, \text { abs }}($ day) & $1.61(0.957)$ & $1.27[0.897-3.80]$ & $1.61(0.957)$ & $1.27[0.897-3.80]$ & $1.61(0.957)$ & $1.27[0.897-3.80]$ \\
\hline$C_{\max , \mathrm{SS}}(\mu \mathrm{g} / \mathrm{mL})$ & $54.9(15.9)$ & $53.9[30.9-82.4]$ & $58.1(16.5)$ & $57.0[33.6-85.9]$ & $66.8(17.7)$ & $65.9[40.4-97.7]$ \\
\hline$t_{\max , \mathrm{SS}}($ day $)$ & $2.51(0.29)$ & $2.50[2.25-3.00]$ & $3.76(0.71)$ & $3.50[3.00-5.25]$ & $4.99(1.35)$ & $4.50[2.50-10.0]$ \\
\hline$C_{\text {trough, } \mathrm{SS}}(\mu \mathrm{g} / \mathrm{mL})$ & $51.1(15.3)$ & 49.9 [28.4-78.7] & $46.7(14.9)$ & $45.6[24.9-75.0]$ & $38.3(14.3)$ & $36.5[17.7-64.7]$ \\
\hline$C_{\max , \mathrm{SS}} / C_{\text {trough, } \mathrm{SS}}(-)$ & $1.08(0.03)$ & 1.07 [1.03-1.15] & $1.26(0.12)$ & 1.24 [1.12-1.49] & $1.85(0.46)$ & 1.74 [1.36-2.85] \\
\hline $\mathrm{AUC}_{\mathrm{ss}, \tau}(\mu \mathrm{g} \times$ day $/ \mathrm{mL})$ & $375(108)$ & $366[211-568]$ & 749 (219) & 733 [423-1135] & 1499 (439) & 1465 [845-2271] \\
\hline$C_{\mathrm{av}, \mathrm{SS}}(\mu \mathrm{g} / \mathrm{mL})$ & $53.5(15.7)$ & $52.3[30.2-81.1]$ & $53.5(15.7)$ & $52.3[30.2-81.1]$ & $53.5(15.7)$ & $52.3[30.2-81.1]$ \\
\hline
\end{tabular}

$N=381$, AUCss, $\tau$ steady-state AUC over dosing interval $\tau$, with $\tau=1$ for $\mathrm{QW}, 2$ for $\mathrm{Q} 2 \mathrm{~W}$, or 4 weeks for $\mathrm{Q} 4 \mathrm{~W}, C_{a v, S S}$ steady-state average concentrations, $C_{\max , S S}$ maximum concentration at steady state, $C_{\text {trough,SS }}$ trough/minimum concentration at steady state, $P K$ pharmacokinetic, $Q W$ every week, $Q 2 W$ every 2 weeks, $Q 4 W$ every 4 weeks, $S D$ standard deviation, $t_{1 / 2}$ elimination half-life, $t_{1 / 2, a b s}$ absorption half-life, $t_{m a x, S S}$ time to reach maximum concentration at steady state
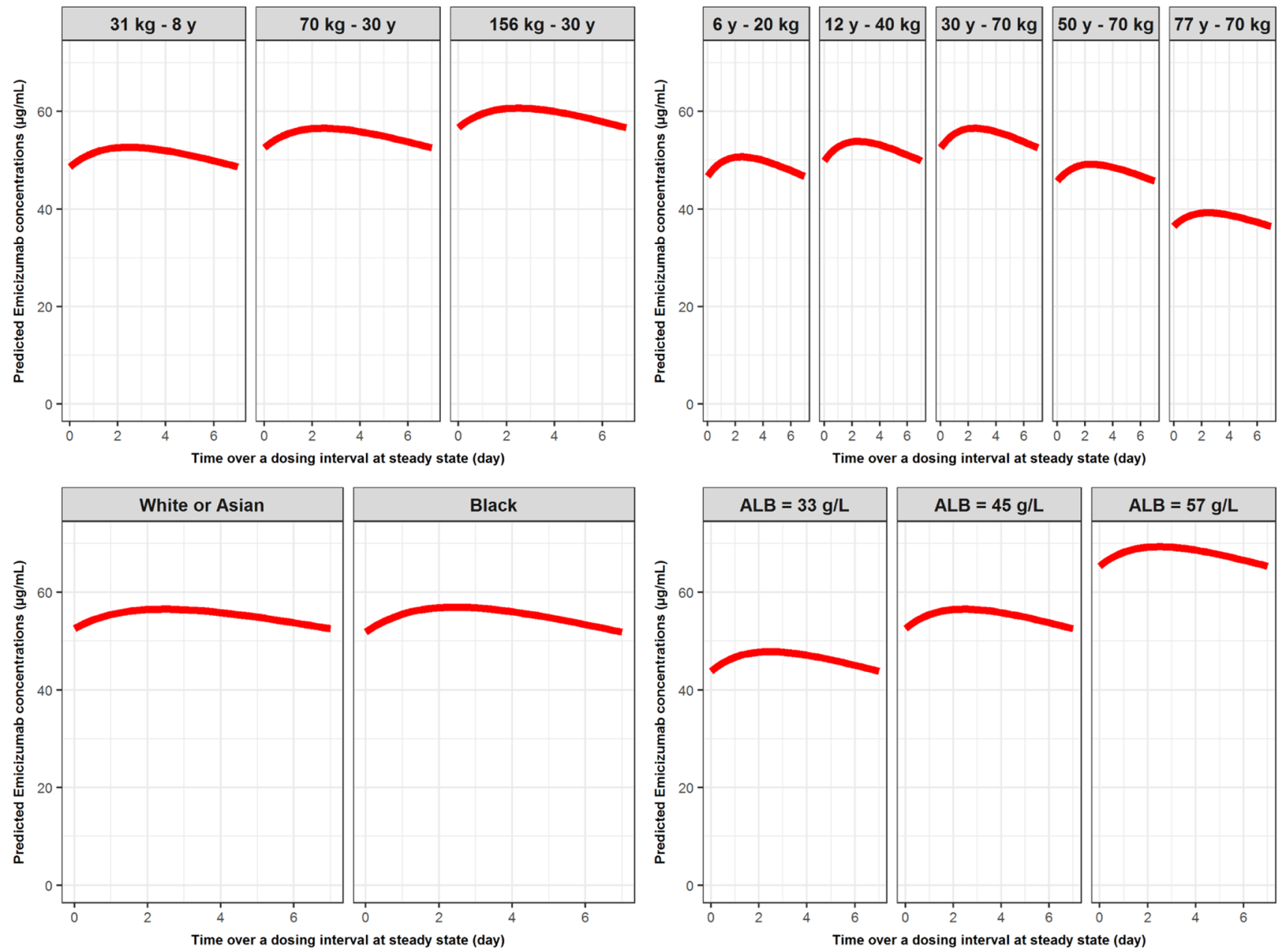

Fig. 4 Illustration of the impact of body weight (top left), age (top right), race (bottom left), and albumin (ALB) level (bottom right) on the predicted pharmacokinetic concentration profile at steady state following $1.5 \mathrm{mg} / \mathrm{kg}$ every week subcutaneously. Top left: predictions performed for white persons with hemophilia A (PwHA) with

an ALB level of $45 \mathrm{~g} / \mathrm{L}$. Top right: predictions performed for white PwHA with an ALB level of $45 \mathrm{~g} / \mathrm{L}$. Bottom left: predictions performed for 30-year-old PwHA weighing $70 \mathrm{~kg}$ with an ALB level of $45 \mathrm{~g} / \mathrm{L}$. Bottom right: predictions performed for white 30-year-old PwHA weighing $70 \mathrm{~kg}$. $y$ years 


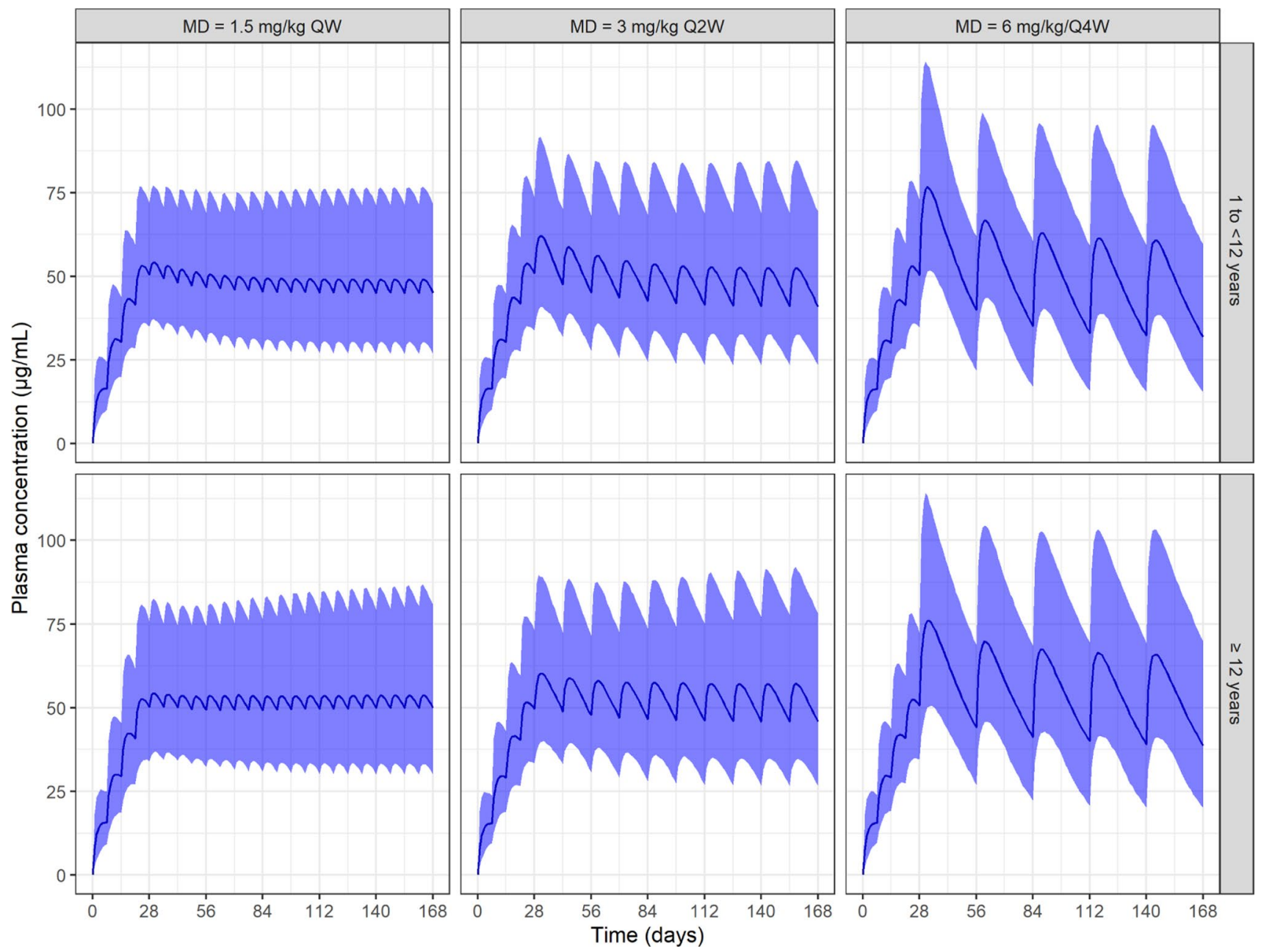

Fig. 5 Predicted pharmacokinetic time course in persons with hemophilia A (PwHA) 1 to $<12$ years or $\geq 12$ years with respect to the three different maintenance doses: $1.5 \mathrm{mg} / \mathrm{kg}$ every week $(\mathrm{QW})$,
$3 \mathrm{mg} / \mathrm{kg}$ every 2 weeks (Q2W), or $6 \mathrm{mg} / \mathrm{kg}$ every 4 weeks (Q4W). Blue curve: median of predictions. Blue area: $90 \%$ prediction intervals. $M D$ maintenance doses or the PwHA status (with or without FVIII inhibitors). This qualified its use for simulation.

Mild hepatic or renal impairment had no impact on the PK parameters (Tables S1-S3 of the ESM). No conclusions on the impact of moderate or severe impairment could be drawn owing to the very small sample size, and because no participants had severe impairment.

\subsection{Pharmacokinetic Model-Based Simulations}

The individual secondary PK parameters are summarized in Table 5. The estimated mean (standard deviation) elimination $t_{1 / 2}$ and absorption $\mathrm{t}_{1 / 2}$ for emicizumab were 26.8 days ( 9.16 days) and 1.61 days ( 0.957 days), respectively.
The impact of BW, age, race (i.e., black vs other races), and ALB on the popPK profiles at steady state are illustrated in Fig. 4 for PwHA receiving emicizumab $1.5 \mathrm{mg} / \mathrm{kg} \mathrm{QW}$ SC. Similar graphs for a dose of $3 \mathrm{mg} / \mathrm{kg}$ Q2W SC or $6 \mathrm{mg} /$ $\mathrm{kg}$ Q4W SC are depicted in Figs. S4 and S5 of the ESM.

Low variation due to BW impact on the level of PK concentration at steady state was predicted, within a range of $50-60 \mu \mathrm{g} / \mathrm{mL}$, as is expected with weight-based dosing. The race effect seen in black PwHA on $V / F$ had no impact on the PK profile at steady state; there was negligible reduction in the $C_{\text {trough,SS }}(<2 \%)$ and augmentation of $C_{\max , \mathrm{SS}}(<1 \%)$ for a black PwHA compared with a white or Asian PwHA (assuming other parameters were equivalent: 30 years of age, weight $70 \mathrm{~kg}$, and ALB level of $45 \mathrm{~g} / \mathrm{L}$ ). Compared to that same reference PwHA, with estimated $C_{\mathrm{av}, \mathrm{SS}}$ of $55.1 \mu \mathrm{g} / \mathrm{mL}$, 


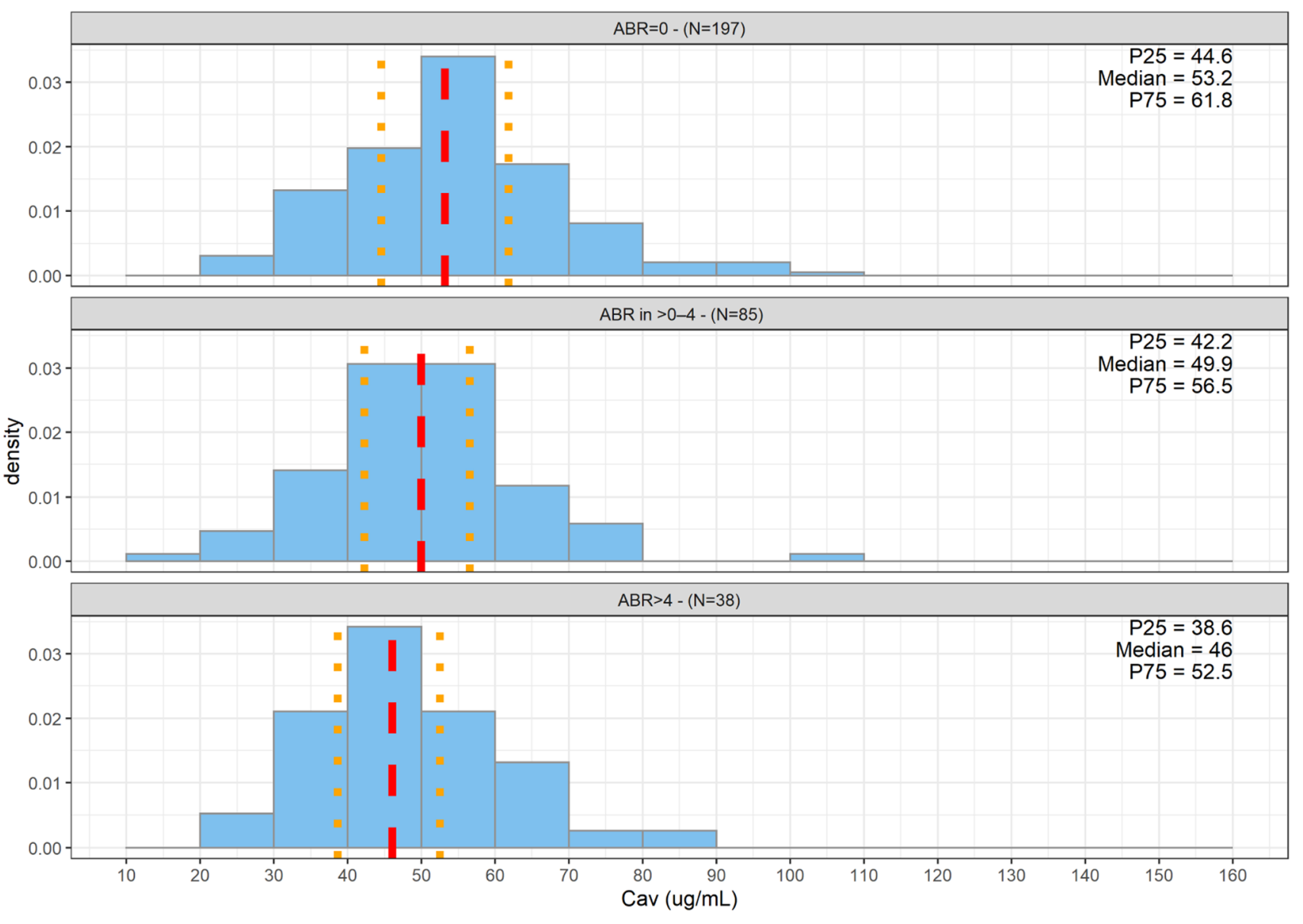

Fig. 6 Distribution of estimated average concentrations $\left(C_{\mathrm{av}}\right)$ by category of annualized bleeding rate (ABR) for persons with hemophilia A from studies HAVEN 1, HAVEN 2, HAVEN 3, and HAVEN 4 (expansion cohort), who received at least 24 weeks of emicizumab treatment. The dotted red line represents the median of the $C_{\mathrm{av}}$ and

a decrease of $16 \%$ in $C_{\mathrm{av}, \mathrm{SS}}$ at a low ALB level of $33 \mathrm{~g} / \mathrm{L}$ $\left(C_{\mathrm{av}, \mathrm{SS}}=46.4 \mu \mathrm{g} / \mathrm{mL}\right)$ and an increase of $23 \%$ at a high ALB level of $57 \mathrm{~g} / \mathrm{L}\left(C_{\mathrm{av}, S \mathrm{SS}}=68.0 \mu \mathrm{g} / \mathrm{mL}\right)$ were predicted, as well as a $31 \%$ reduction in steady-state exposure in the case of a 77-year-old PwHA. This latter result was supported by the observation that PK profiles had a tendency toward lower concentrations in older age PwHA (data not shown).

Predicted PK profiles with respect to pediatric PwHA $\geq 1$ to $<12$ years and adolescent and adult $\mathrm{PwHA}(\geq 12$ years) for the three different dosing regimens $(\mathrm{QW}, \mathrm{Q} 2 \mathrm{~W}$, and $\mathrm{Q} 4 \mathrm{~W})$ over a period of 6 months of emicizumab administrations are reported in Fig. 5. Negligible differences were observed in the PK profiles between PwHA aged 1 to $<12$ years and older PwHA. As expected, larger fluctuation in the concentrations over the dosing interval was observed with a less frequent dosing regimen, although the average concentration remained constant across regimen groups. the orange dotted lines the 25th (P25) and 75th (P75) percentiles of observation, respectively. Distributions are represented as density histograms so that the area of each rectangle equals the relative frequency of the corresponding class, and the area of the entire histogram equals 1

\subsection{Exploratory Exposure-Efficacy Relationships}

A total of 320 PwHA were treated for at least 24 weeks. As described in several studies [11-14], clinically meaningful prevention of bleeding was observed for PwHA receiving QW, Q2W, or Q4W emicizumab administrations, with a large proportion of PwHA with zero treated bleeds (61.6\%). There was a limited proportion of PwHA with an ABR $>4$ (11.9\%). Only 11 patients in the HAVEN studies were uptitrated to $3 \mathrm{mg} / \mathrm{kg}$ QW.

Figure 6 displays the distributions of the estimated $C_{\text {av }}$ by ABR category; further splits per dosing regimens (Fig. S6 of the ESM) and per PwHA status (with or without FVIII inhibitors, Figs. S7 and S8 of the ESM) are depicted in the ESM. The exposure distributions by ABR category largely overlapped. However, a very slight, but consistent, decrease in exposure was observed with an increase of ABR category severity, leading to a slightly lower median emicizumab $C_{\mathrm{av}}$ 
in the $\mathrm{ABR}$ of $>4$, independent of dosing regimen or PwHA status.

\section{Discussion}

The PK characteristics of emicizumab following SC administrations in PwHA with or without FVIII inhibitors were investigated using a popPK approach. The popPK model, developed using a database of 383 PwHA aged between 1.22 and 77.0 years, was a one-compartment model with first-order absorption and elimination processes. The pharmacokinetics of antibodies is usually described by a twocompartment model; however, the SC formulation of emicizumab may imply an initial distribution phase masked by the absorption phase, only the terminal disposition phase therefore being visible. The final popPK model relies on dose- and time-independent PK parameters; they were accurately estimated and the diagnostic plots did not indicate any major unexpected deficiencies in the model. Because of the low magnitude of the proportional errors (14.6\%) and the low within-person variability in the observed $C_{\text {trough,Ss }}$ over time, no inter-occasion variabilities were deemed necessary to be included [33]. The visual predictive checks per study and per dosing regimen (Fig. 3) demonstrated the good performance of the model in describing the pharmacokinetics of emicizumab in pediatric, adolescent, and adult PwHA, whatever the dosing regimen.

The absorption and elimination $t_{1 / 2}$ were estimated at 1.61 days and 26.8 days, respectively. This elimination is slightly longer than for classical humanized immunoglobulin $\mathrm{G}$ antibodies [34], and may be a consequence of a reduced isoelectric point from the antibody engineering [7, 35].

A positive correlation between BW and both CL/F and V/F was estimated, likely representing the catabolism of the antibody by the reticuloendothelial system in reticular connective tissues widely distributed throughout the body [36]. Weight-based dosing showed rather similar steady-state exposures across a large range of BWs (Fig. 4). For $V / F$, the BW exponent matched the classical allometric exponent of 1 ; for $\mathrm{CL} / F$, a higher exponent (0.91) than the classical 0.75 value was found. This leads to lower clearance in younger children compared to allometry, probably owing to a maturation effect.

A lower $V / F(-22 \%)$ in black PwHA compared with white PwHA was detected, although no clear explanation for this finding has currently been found. It has, however, no obvious impact on the steady-state exposure, as illustrated in Fig. 4.

A positive correlation between ALB and CL/ $F$ was also detected, leading for instance to a $16 \%$ reduction in exposure in a PwHA with an ALB level of $33 \mathrm{~g} / \mathrm{L}$ compared to the typical $45 \mathrm{~g} / \mathrm{L}$ median value. Albumin and immunoglobulin $\mathrm{G}$ are both recycled by the neonatal Fc receptors; ALB in plasma could therefore be a surrogate for the activity of neonatal Fc receptor protein in the individuals [34], explaining the detected correlation between ALB and CL/F. Nevertheless, for the PwHA from the four phase III HAVEN studies, the lower exposure with low ALB did not translate into lower efficacy, with, overall, observed ABRs close to 0 in both groups of PwHA with ALB at baseline $\geq 45 \mathrm{~g} / \mathrm{L}$ $(N=205)$ and $<45 \mathrm{~g} / \mathrm{L}(N=159)$.

The apparent bioavailability gradually decreased for PwHA aged older than 30 years, leading to a predicted steady-state exposure decrease of $31 \%$ in a 77 -year-old PwHA compared to a 30-year-old PwHA having an $F$ of 1 . This may reflect a potential age-related decrease in hypodermis thickness [37] or reduced neonatal Fc receptor expressing cells and/or lymphatic drainage; all of these are key players in SC bioavailability of therapeutic antibodies [38, 39]. Nevertheless, the lower exposure in elderly individuals does not impair efficacy, with, overall, observed ABRs close to 0 in both elderly individuals (age $\geq 65$ years, $N=13$ ) and other (agd $<65$ years, $N=351$ ) PwHA.

For PwHA aged 1-30 years, no age effect on primary PK parameters was found once the body size effect on CL/F and $V / F$ was accounted for in the model. Emicizumab PK characteristics in pediatric PwHA aged younger than 1 year were also investigated, taking into account the impact of organ maturation on the drug disposition [40]; this will be reported in a future publication.

None of the other covariates tested either statistically (i.e., FVIII inhibitor or non-inhibitor status, body mass index, body surface area) or graphically (National Cancer Institute classification for hepatic impairment, renal function) was found to further explain the PK variability of emicizumab. At the time of the popPK model development, four PwHA had anti-emicizumab antibodies; only one of them experienced an associated decline in the emicizumab PK profile and was excluded from the model development.

A minor trend toward a reduction in the ABR with increasing median emicizumab $C_{\mathrm{av}}$ was observed, suggesting that the plateau of the exposure-response relationship has been reached but that reducing emicizumab exposure to lower levels may potentially increase the risk of bleeding. The three maintenance doses of $1.5 \mathrm{mg} / \mathrm{kg}$ QW SC, $3 \mathrm{mg} /$ $\mathrm{kg}$ Q2W SC, and $6 \mathrm{mg} / \mathrm{kg}$ Q4W SC are predicted to generate similar emicizumab $C_{\mathrm{av}}$ associated with a clinically meaningful reduction in the ABR. The relationship between emicizumab exposure and the ABR explored in this article, as well as the lack of impact of age and ALB on efficacy were further confirmed via exposure-efficacy relationship modeling [41] (to be published separately). 


\section{Conclusions}

Emicizumab pharmacokinetics was characterized in adults, adolescents (age $\geq 12$ years and $<18$ years), and children (age $>1$ year and $<12$ years) with hemophilia A with or without FVIII inhibitors. Body weight is an important predictor of emicizumab exposure, justifying the weight-based dosing. The three dosing regimens: $3 \mathrm{mg} / \mathrm{kg}$ QW SC for 4 weeks followed by $1.5 \mathrm{mg} / \mathrm{kg} \mathrm{QW}, 3 \mathrm{mg} / \mathrm{kg}$ Q2W, or $6 \mathrm{mg} /$ $\mathrm{kg}$ Q4W SC, are predicted to generate similar emicizumab exposures associated with clinically meaningful prevention of bleeding.

Acknowledgements The authors thank Dr. Koichiro Yoneyama for his valuable comments and suggestions during the manuscript preparation. This study was funded by F. Hoffmann-La Roche, Ltd.

\section{Compliance with Ethical Standards}

Funding This study was funded by F. Hoffmann-La Roche Ltd. Open access publication of this manuscript was sponsored by F. HoffmannLa Roche, Ltd.

Conflict of interest Sylvie Retout, Christophe Schmitt, Claire Petry, François Mercier, and Nicolas Frey are employees of F. Hoffmann-La Roche, Ltd. Sylvie Retout, Christophe Schmitt, and Nicolas Frey hold stocks/stock options with F. Hoffmann-La Roche, Ltd.

Ethics approval All procedures performed in studies involving human participants were approved by the institutional review board at each site and in accordance with the 1964 Helsinki Declaration and its later amendments or comparable ethical standards.

Consent to participate Informed consent was obtained from all individual participants included in the studies discussed.

Data sharing Qualified researchers may request access to individual patient-level data through the clinical study data request platform (www.clinicalstudydatarequest.com). Further details on Roche's criteria for eligible studies are available here: https://clinicalstudydatareq uest.com/Study-Sponsors/Study-Sponsors-Roche.aspx. For further details on Roche's Global Policy on the Sharing of Clinical Information and how to request access to related clinical study documents, go to: https://www.roche.com/research_and_development/who_we_are_ how_we_work/clinical_trials/our_commitment to data_sharing.htm.

Open Access This article is licensed under a Creative Commons Attribution-NonCommercial 4.0 International License, which permits any non-commercial use, sharing, adaptation, distribution and reproduction in any medium or format, as long as you give appropriate credit to the original author(s) and the source, provide a link to the Creative Commons licence, and indicate if changes were made. The images or other third party material in this article are included in the article's Creative Commons licence, unless indicated otherwise in a credit line to the material. If material is not included in the article's Creative Commons licence and your intended use is not permitted by statutory regulation or exceeds the permitted use, you will need to obtain permission directly from the copyright holder. To view a copy of this licence, visit http://creativecommons.org/licenses/by-nc/4.0/.

\section{References}

1. Manco-Johnson MJ, Abshire TC, Shapiro AD, Riske B, Hacker MR, Kilcoyne R, et al. Prophylaxis versus episodic treatment to prevent joint disease in boys with severe hemophilia. N Engl J Med. 2007;357(6):535-44. https://doi.org/10.1056/NEJMoa0676 59.

2. Ljung $\mathrm{R}$. The risk associated with indwelling catheters in children with haemophilia. Br J Haematol. 2007;138(5):580-6. https://doi. org/10.1111/j.1365-2141.2007.06703.x.

3. Franchini M, Tagliaferri A, Mengoli C, Cruciani M. Cumulative inhibitor incidence in previously untreated patients with severe hemophilia A treated with plasma-derived versus recombinant factor VIII concentrates: a critical systematic review. Crit Rev Oncol Hematol. 2012;81(1):82-93. https://doi.org/10.1016/j.critr evonc.2011.01.002.

4. Leissinger CA, Konkle BA, Antunes SV. Prevention of bleeding in hemophilia patients with high-titer inhibitors. Expert Rev Hematol. 2015;8(3):375-82. https://doi.org/10.1586/17474 086.2015.1036733.

5. Konkle BA, Ebbesen LS, Erhardtsen E, Bianco RP, Lissitchkov T, Rusen L, et al. Randomized, prospective clinical trial of recombinant factor VIIa for secondary prophylaxis in hemophilia patients with inhibitors. J Thromb Haemost. 2007;5(9):1904-13. https:// doi.org/10.1111/j.1538-7836.2007.02663.x.

6. Kitazawa T, Esaki K, Tachibana T, Ishii S, Soeda T, Muto A, et al. Factor VIIIa-mimetic cofactor activity of a bispecific antibody to factors IX/IXa and X/Xa, emicizumab, depends on its ability to bridge the antigens. Thromb Haemost. 2017;117(7):1348-57. https://doi.org/10.1160/TH17-01-0030.

7. Sampei Z, Igawa T, Soeda T, Okuyama-Nishida Y, Moriyama $\mathrm{C}$, Wakabayashi $\mathrm{T}$, et al. Identification and multidimensional optimization of an asymmetric bispecific IgG antibody mimicking the function of factor VIII cofactor activity. PLOS ONE. 2013;8(2):e57479. https://doi.org/10.1371/journal.pone.0057479.

8. Kotani N, Yoneyama K, Kawakami N, Shimuta T, Fukase H, Kawanishi T. Relative and absolute bioavailability study of emicizumab to bridge drug products and subcutaneous injection sites in healthy volunteers. Clin Pharmacol Drug Dev. 2019;8(6):702-12. https://doi.org/10.1002/cpdd.617.

9. Uchida N, Sambe T, Yoneyama K, Fukazawa N, Kawanishi T, Kobayashi S, et al. A first-in-human phase 1 study of ACE910, a novel factor VIII-mimetic bispecific antibody, in healthy subjects. Blood. 2016;127(13):1633-41. https://doi.org/10.1182/blood -2015-06-650226.

10. Valentino LA, Pipe SW, Collins PW, Blanchette VS, Berntorp E, Fischer K, et al. Association of peak factor VIII levels and area under the curve with bleeding in patients with haemophilia $\mathrm{A}$ on every third day pharmacokinetic-guided prophylaxis. Haemophilia. 2016;22(4):514-20. https://doi.org/10.1111/hae.12905.

11. Oldenburg J, Mahlangu JN, Kim B, Schmitt C, Callaghan MU, Young G, et al. Emicizumab prophylaxis in hemophilia A with inhibitors. N Engl J Med. 2017;377(9):809-18. https://doi. org/10.1056/NEJMoa1703068.

12. Young G, Liesner R, Chang T, Sidonio R, Oldenburg J, JimenezYuste $\mathrm{V}$, et al. A multicenter, open-label phase 3 study of emicizumab prophylaxis in children with hemophilia A with inhibitors. Blood. 2019;134(24):2127-38. https://doi.org/10.1182/blood 2019001869

13. Mahlangu J, Oldenburg J, Paz-Priel I, Negrier C, Niggli M, Mancuso ME, et al. Emicizumab prophylaxis in patients who have hemophilia A without inhibitors. N Engl J Med. 2018;379(9):81122. https://doi.org/10.1056/NEJMoa1803550.

14. Pipe SW, Shima M, Lehle M, Shapiro A, Chebon S, Fukutake $\mathrm{K}$, et al. Efficacy, safety, and pharmacokinetics of emicizumab 
prophylaxis given every 4 weeks in people with haemophilia $\mathrm{A}$ (HAVEN 4): a multicentre, open-label, non-randomised phase 3 study. Lancet Haematol. 2019;6(6):e295-305. https://doi. org/10.1016/S2352-3026(19)30054-7.

15. Yoneyama K, Schmitt C, Kotani N, Levy GG, Kasai R, Iida S, et al. A pharmacometric approach to substitute for a conventional dose-finding study in rare diseases: example of phase III dose selection for emicizumab in hemophilia A. Clin Pharmacokinet. 2018;57(9):1123-34. https://doi.org/10.1007/s40262-017-0616-3.

16. Blanchette VS, Key NS, Ljung LR, Manco-Johnson MJ, van den Berg HM, Srivastava A, et al. Definitions in hemophilia: communication from the SSC of the ISTH. J Thromb Haemost. 2014;12(11):1935-9. https://doi.org/10.1111/jth.12672.

17. Shima M, Hanabusa H, Taki M, Matsushita T, Sato T, Fukutake $\mathrm{K}$, et al. Factor VIII-mimetic function of humanized bispecific antibody in hemophilia A. N Engl J Med. 2016;374(21):2044-53. https://doi.org/10.1056/NEJMoa1511769.

18. Shima M, Hanabusa H, Taki M, Matsushita T, Sato T, Fukutake $\mathrm{K}$, et al. Long-term safety and efficacy of emicizumab in a phase $1 / 2$ study in patients with hemophilia A with or without inhibitors. Blood Adv. 2017;1(22):1891-9. https://doi.org/10.1182/blood advances.2017006684.

19. Mandema JW, Verotta D, Sheiner LB. Building population pharmacokinetic-pharmacodynamic models. I. Models for covariate effects. J Pharmacokinet Biopharm. 1992;20(5):511-28.

20. Burnham KP, Anderson DR, Huyvaert KP. AIC model selection and multimodel inference in behavioral ecology: some background, observations, and comparisons. Behav Ecol Sociobiol. 2010;65(1):23-5.

21. Frey N, Grange S, Woodworth T. Population pharmacokinetic analysis of tocilizumab in patients with rheumatoid arthritis. J Clin Pharmacol. 2010;50(7):754-66. https://doi.org/10.1177/00912 70009350623.

22. Harrell F. Regression modeling strategies. New York: Springer; 2001.

23. Gastonguay MR. A full model estimation approach for covariate effects: interference based on clinical importance and estimation precision. AAPS J. 2004;6(1):W4354.

24. Ravva P, Gastonguay MR, Tensfeldt TG, Faessel HM. Population pharmacokinetic analysis of varenicline in adult smokers. Br J Clin Pharmacol. 2009;68(5):669-81. https://doi.org/10.111 $1 / \mathrm{j} .1365-2125.2009 .03520 . x$.

25. Gastonguay MR. A-16 full covariate models as an alternative to methods relying on statistical significance for inferences about covariate effects: a review of methodology and 42 case studies. 2011. Available from: https://www.page-meeting.org/?abstr act $=2229 \#$. Accessed 14 May 2020.

26. National Kidney Foundation. K/DOQI clinical practice guidelines for chronic kidney disease: evaluation, classification, and stratification. Am J Kidney Dis. 2002;39(2 Suppl. 1):S1-266.

27. Cockcroft DW, Gault MH. Prediction of creatinine clearance from serum creatinine. Nephron. 1976;16(1):31-41. https://doi. org/10.1159/000180580.

28. Schwartz GJ, Work DF. Measurement and estimation of GFR in children and adolescents. Clin J Am Soc Nephrol. 2009;4(11):1832-43. https://doi.org/10.2215/cjn.01640309.
29. Patel H, Egorin MJ, Remick SC, Mulkerin D, Takimoto CHM, Doroshow JH, et al. Comparison of Child-Pugh (CP) criteria and $\mathrm{NCI}$ organ dysfunction working group (NCI-ODWG) criteria for hepatic dysfunction (HD): implications for chemotherapy dosing. J Clin Oncol. 2004;22(14_Suppl.):6051. https://doi.org/10.1200/ jco.2004.22.90140.6051.

30. Bergstrand M, Hooker AC, Wallin JE, Karlsson MO. Prediction-corrected visual predictive checks for diagnosing nonlinear mixed-effects models. AAPS J. 2011;13(2):143-51. https://doi. org/10.1208/s12248-011-9255-z.

31. Savic RM, Karlsson MO. Importance of shrinkage in empirical bayes estimates for diagnostics: problems and solutions. AAPS J. 2009;11(3):558-69. https://doi.org/10.1208/s12248-009-9133-0.

32. Andropoulos DB. Appendix B, pediatric normal laboratory values. In: Gregory GA, Andropoulos DB, editors. Gregory's pediatric anesthesia. 5th ed. Hoboken, New Jersey: Wiley-Blackwell, John Wiley \& Sons; 2012.

33. Karlsson MO, Sheiner LB. The importance of modeling interoccasion variability in population pharmacokinetic analyses. J Pharmacokinet Biopharm. 1993;21(6):735-50. https://doi.org/10.1007/ bf01113502.

34. Dostalek M, Gardner I, Gurbaxani BM, Rose RH, Chetty M. Pharmacokinetics, pharmacodynamics and physiologically-based pharmacokinetic modelling of monoclonal antibodies. Clin Pharmacokinet. 2013;52(2):83-124. https://doi.org/10.1007/s4026 2-012-0027-4

35. Igawa $\mathrm{T}$, Tsunoda $\mathrm{H}$, Tachibana $\mathrm{T}$, Maeda A, Mimoto F, Moriyama $\mathrm{C}$, et al. Reduced elimination of IgG antibodies by engineering the variable region. Protein Eng Des Sel. 2010;23(5):385-92. https://doi.org/10.1093/protein/gzq009.

36. Tabrizi MA, Tseng CM, Roskos LK. Elimination mechanisms of therapeutic monoclonal antibodies. Drug Discov Today. 2006;11(1-2):81-8. https://doi.org/10.1016/s1359 $-6446(05) 03638-x$.

37. Ryman JT, Meibohm B. Pharmacokinetics of monoclonal antibodies. CPT Pharmacometrics Syst Pharmacol. 2017;6(9):576-88. https://doi.org/10.1002/psp4.12224.

38. Deng R, Meng YG, Hoyte K, Lutman J, Lu Y, Iyer S, et al. Subcutaneous bioavailability of therapeutic antibodies as a function of FcRn binding affinity in mice. mAbs. 2012;4(1):101-9. https ://doi.org/10.4161/mabs.4.1.18543.

39. Richter WF, Jacobsen B. Subcutaneous absorption of biotherapeutics: knowns and unknowns. Drug Metab Dispos. 2014;42(11):1881-9. https://doi.org/10.1124/dmd.114.059238.

40. Retout S, Grimm H-P, Petry C, Schmitt C, Frey N. A modelbased extrapolation enabled labelling of emicizumab in haemophilia A paediatric patients $<1$ year old despite lack of clinical data [abstract no. 9089]. 2019. p. 28. http://www.page-meeti ng.org/?abstract $=9089$. Accessed 20 May 2020.

41. Jonsson F, Schmitt C, Petry C, Mercier F, Frey N, Retout S. Exposure-response modeling of emicizumab for the prophylaxis of bleeding in haemophilia A patients with and without inhibitors against factor VIII [abstract no. PB0325]. Res Pract Thromb Haemostasis. 2019;3(S1):1-891. 